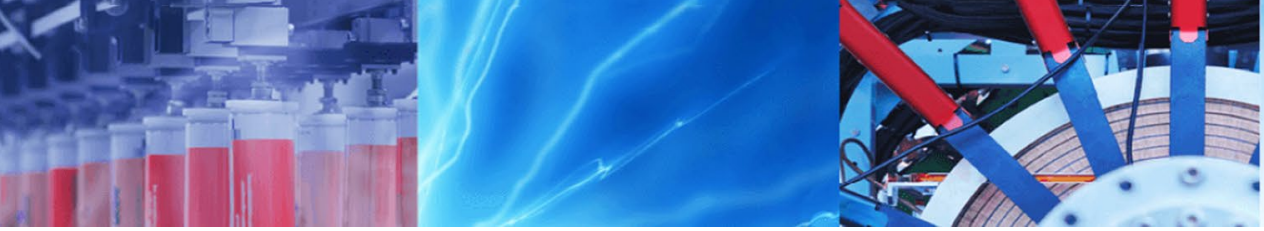

Research Article

\title{
Radar seeker performance evaluation based on information fusion method
}

\author{
Xinghai Liu ${ }^{1}$ - Jian Yang ${ }^{1,2} \cdot$ Bo Hou $^{1} \cdot$ Jian Lu$^{1} \cdot$ Zhicheng Yao $^{1}$
}

Received: 15 December 2019 / Accepted: 12 March 2020 / Published online: 17 March 2020

(c) Springer Nature Switzerland AG 2020

\begin{abstract}
Radar seeker is responsible for transmitting the dynamic position information of the target back to the weapon control center in the guidance phase. It is of great engineering significance to evaluate the anti-jamming ability of radar seeker in the anti-jamming environment Aimed at obtaining more reasonable evaluation result, an evaluation method based on information fusion is proposed against the background of angle measurement performance in this paper. Radar seeker performance test have many stages with different characteristics. Information fusion has advantages in radar performance evaluation which takes advantage of measured data obtained in different stages rather than process them respectively. Furthermore, we studied two specific issues in the information-fusion stage. The first problem is about consistency testing between different information using K-L information distance measurement, which is the premise of information fusion. The second problem is about the information weights calculation by PLT criterion, which can take sample size into account. Information fusion can make full use of the multi-source test information to obtain more reliable evaluation conclusion. Experimental results prove the effectiveness of the proposed method.
\end{abstract}

Keywords Angle measurement · Performance evaluation · Information fusion · Consistency testing

\section{Introduction}

Radar seeker is the eye of guided weapon, which has the function of target detection and location. In the process of attacking the target, the radar seeker is responsible for transmitting the dynamic position information of the target back to the weapon control center [1, 2].From the point of view of protecting targets, many EMI equipment are developing to weaken the guidance ability of radar seeker [3, 4]. It is of great engineering significance to evaluate the anti-jamming ability of radar seeker in the antijamming environment $[5,6]$. A performance evaluation method for ISAR under jamming condition is introduced in literature [7]. Evaluation method for adaptive detection performance of fluctuating radar targets is studied in literature [8].Target detection performance of spectrum sharing MIMO radars is analyzed in literature [9]. An effective nonlinear data processing method is proposed in the literature $[10,11]$ and this method is useful in target perception algorithm based on recurrent neural network. Existing evaluation schemes have not fully utilized test data [12]. To solve this problem, we propose a performance evaluation scheme based on information fusion.

Performance evaluation is a systematic project, and a complete evaluation index system is the basis of performance evaluation. Effective evaluation index can accurately measure the characteristics of the research object. The performance evaluation indicator system is to integrate a series of effective indicators into a complete framework. Two important criteria, integrity and conciseness, should be abided by in the construction of index system. These two criteria make the index system not

Xinghai Liu, 2302975296@qq.com | ${ }^{1}$ Rocket Force University of Engineering, Xi'an 710025, China. ${ }^{2}$ School of Electronic Engineering, Xidian University, Xi'an 710071, China. 
only measure the overall performance of the research object, but also have no redundancy [13]. Radar seeker performance evaluation involves many experimental stages. Common index information can be derived from test data in each stage. On the other hand, considering test environment and method varies in different stages, test data in each stage have different information value [14]. Based on these evaluation characteristics, we can use information fusion method to make greater use of test data in different test stage to obtain more accurate and reliable evaluation conclusion.

In most simultaneous interpreting processes, the observation dataset comes from different sources, such as different sensors or different test environments. On the basis of estimation theory and information fusion theory, parameter fusion estimation studies how to fully mine and utilize the effective information in different data sets. At present, the research and application of parameter fusion estimation are very extensive in the fields of target tracking, situation estimation and risk decision $[15,16]$. Parameter fusion estimation algorithm is based on fusion structure. According to the distribution characteristics of fusion center, the common fusion structures can be divided into two basic structures: distributed fusion and central fusion. In distributed fusion, each data set is preprocessed by different local fusion centers, and then the feature level data is sent to the top-level nodes for centralized fusion processing. There is no local processor in the centralized fusion structure, only a fusion center for analyzing the original test data $[17,18]$. There are some problems in the information fusion application progress, such as information conflict management, information weight distribution and so on. In view of these problems, scholars also put forward corresponding solutions. HOBW method for solving the nonlinear and unsteady process is introduced in the literature [19].A fuzzy information infusion method is proposed in Literature [20]. The divergence of multi sensors information fusion filter is studied in Literature [21]. Conflict measurement method in information fusion is proposed in Literature [22]. Dynamic weights allocation method according to uncertain evaluation information is proposed in literature [23]. Weighted K-L information measures is used to allocate information weight Literature [24]. Weighted matrix completion and recovery method with prior subspace information is introduced in Literature [25].These research results provide good method for the information fusion application in performance evaluation. We have proposed an information fusion evaluation method in literature [26]. In this paper, we focused on index weight and information weigh empowerment method and applied this method in radar seeker performance evaluation. Based on these research results, we further examine some key issues in information fusion assessment.

Aimed at obtaining more reasonable evaluation result, an evaluation method based on information fusion is proposed against the background of angle measurement performance in this paper. Furthermore, we studied two specific issues in the information-fusion stage. The first one is about modeling the difference of performance influencing factors in different experimental environments. The second one is about consistency testing and information weight empowerment method between different information. The remainder of this paper is organized as follows. The characteristics of radar performance test and the advantages of fusion evaluation are introduced in Sect. 2. Information consistency evaluation method based on $\mathrm{K}-\mathrm{L}$ information distance measurement and information weight assignment method based on PLT criterion is introduced in Sect. 3. Section 4 illustrates the validity of the method mentioned in this paper with a set of test data in simulation and field experiment. Some discussion and conclusion of information-fusion process in radar performance evaluation is presented in Sect. 5 .

\section{Information fusion evaluation}

\subsection{Radar seeker performance test stage}

The radar seeker performance test scheme includes different stages, such as mathematical simulation, hardwarein-the-loop and field test as shown in Fig. 1. Mathematical simulation test refers to the construction of a performance test mathematical model. The model elements include transmitting signal and antenna beam, target and jamming environment simulation, echo signal processing and other processes. On this basis, a computer program is used to complete the functional verification of the radar seeker. Mathematical simulation test is simple and easy to operate. The whole test process can be completed by computer with high repeatability. The disadvantage is that

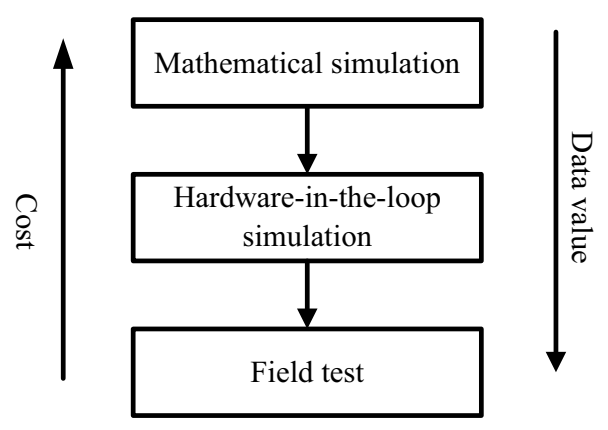

Fig. 1 Characteristics difference in test stages 
it is difficult to establish a mathematical model similar to the actual environment, and the test results can only be used for preliminary functional verification.

Hardware-in-the-loop simulation testing refers to the process that real equipment under simulation test environment. In this process, we use the simulator to generate the target and jamming environment signal needed for system performance testing and then introduce the signal into the tested radar seeker by direct injection or antenna radiation. Finally, we compare the signal processing results and processing expectations of the radar seeker to evaluate its performance. Hardware-in-the-loop simulation is usually carried out in microwave anechoic chamber with electromagnetic shielding function to achieve the purpose that test environment is controllable.

Field test is a practical test in a real electromagnetic environment. The main purpose is to test the radar seeker performance for completing the task under the real clutter and noise background and jamming environment, which can also supplement the low credibility of the simulation test data. The limitation of the field test is that the resource consumption is high and the repeatability is low. The test samples obtained from field tests are small samples and have high credibility and important decision-making reference value.

Referring to literature [26], radar seeker field test experiment is consists of three stages, which include static test, flying experiment and shooting experiment. These three test phases have different characteristics in repeatability and test data value. According to the characteristic of radar seeker performance test stage, we construct an evaluation model as shown in Fig. 2, which is firstly introduced in literature [26]. The test results obtained by different test phases can be regarded as data in different dimensions.

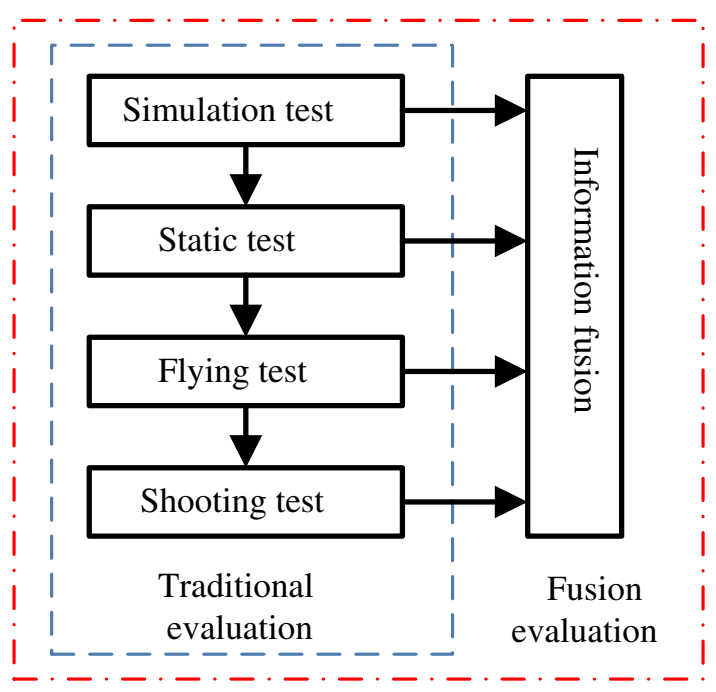

Fig. 2 Characteristics difference in test stages [26]
The basic idea of equivalent estimation is to construct an effective estimation model and obtain a reasonable estimation relationship and then project these data to the same calculation dimension. To accurately establish the difference model of the performance affecting factors in different test environments is the premise of fusion evaluation using equivalent estimation.

\subsection{Angle measurement performance influencing factors}

The significance of microwave anechoic chamber is to form an electromagnetic space with minimum interference and uniform field intensity. This electromagnetic space is called static zone. The platform used to place the measured radar seeker should be static zone as shown in Fig. 3. The main indexes to measure the microwave anechoic chamber performance are static zone size and field intensity uniformity. In fact, there are still different reflected waves paths in the anechoic chamber. The significance of electromagnetic absorbing materials is to absorb these interference waves to the greatest extent and ensure uniform field strength in the static zone.

Under ideal conditions, the static region can be simplified to a circle, and the energy of the four quadrants can be recorded as $Q_{A}, Q_{B}, Q_{C}, Q_{D}$ respectively, as shown in Fig. 4. When the turntable reference error and reflected clutter are considered, the energy of different quadrants varies, which will bring the following errors to the angle measurement [27]:

$\Delta \theta=\left[\left(Q_{A}+Q_{B}\right)-\left(Q_{C}+Q_{D}\right)\right] /\left(Q_{A}+Q_{B}+Q_{C}+Q_{D}\right)$.

Environmental clutter jamming is another important factor affecting radar angle measurement performance. In the hardware-in-the-loop simulation test, the real environment interference signal should be simulated as accurately as possible, so that the test results are effective. The most commonly used mathematical models for clutter simulation include the following three type [28].

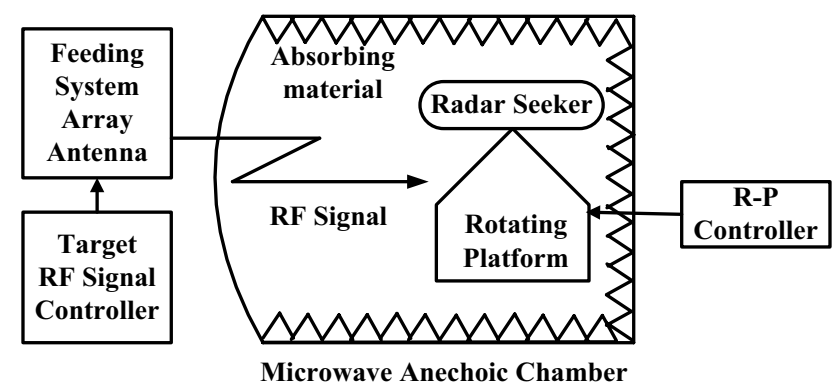

Fig. 3 Microwave anechoic chamber simulation test 


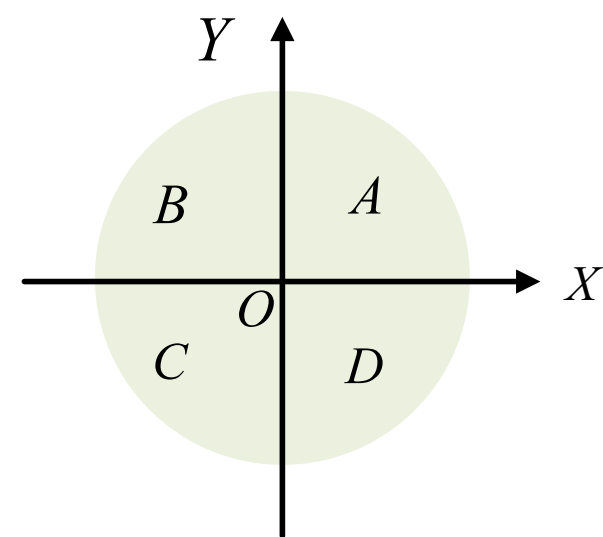

Fig.4 Quiet zone distribution in microwave chamber

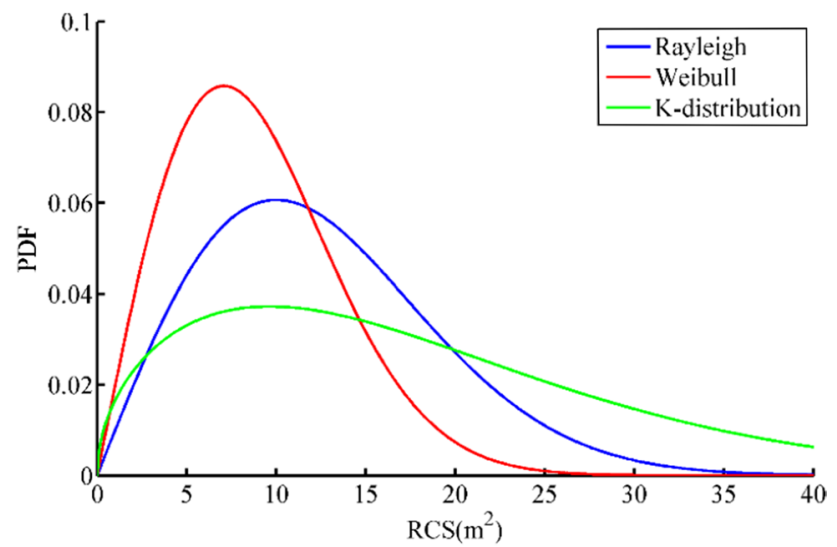

Fig. 5 Clutter RCS distribution model

1. Rayleigh Distribution. RCS PDF is

$$
f(z)=\frac{z}{\sigma^{2}} \exp \left[-\frac{z^{2}}{2 \sigma^{2}}\right]
$$

2. Weibull Distribution. RCS PDF is

$$
f(z)=\left(\frac{z}{q}\right)^{p-1}\left(\frac{p}{q}\right) \exp \left[-\left(\frac{z}{q}\right)^{p}\right] \quad 0<p<2 .
$$

3. K-Distribution. RCS PDF is

$$
K(z, a, v)=\frac{2}{a \Gamma(v+1)}\left(\frac{z}{2 a}\right)^{v+1} K_{v}\left(\frac{z}{a}\right) .
$$

The simulation results of the three models are shown in Fig. 5.

\subsection{Performance evaluation index system and weight empowerment}

In this section, we carried out performance test scheme based on active homing radar seeker and high-power active suppression jamming equipment. A common index system is constructed referring to Radar Test Outline as shown in Fig. 6 and we use the maximizationdeviation method to calculate index weight [26]. The calculation process is as follows.

$\left\{\begin{array}{l}\max z=\sum_{j=1}^{m} d_{j} \omega_{j} \\ \text { s.t. } \sum_{j=1}^{m} \omega_{j}^{2}=1 \quad \omega_{j} \geq 0\end{array}\right.$.

To obtain the optimal solution, the Lagrange equation is constructed as follows:

$L(\omega, \xi)=\sum_{j=1}^{m} d_{j} \omega_{j}+\frac{\xi}{2}\left(\sum_{j=1}^{m} \omega_{j}^{2}-1\right)$.

Then, the partial derivative of $L$ is obtained.

Fig. 6 Performance evaluation index system

\section{Seeker guidance hit probability}

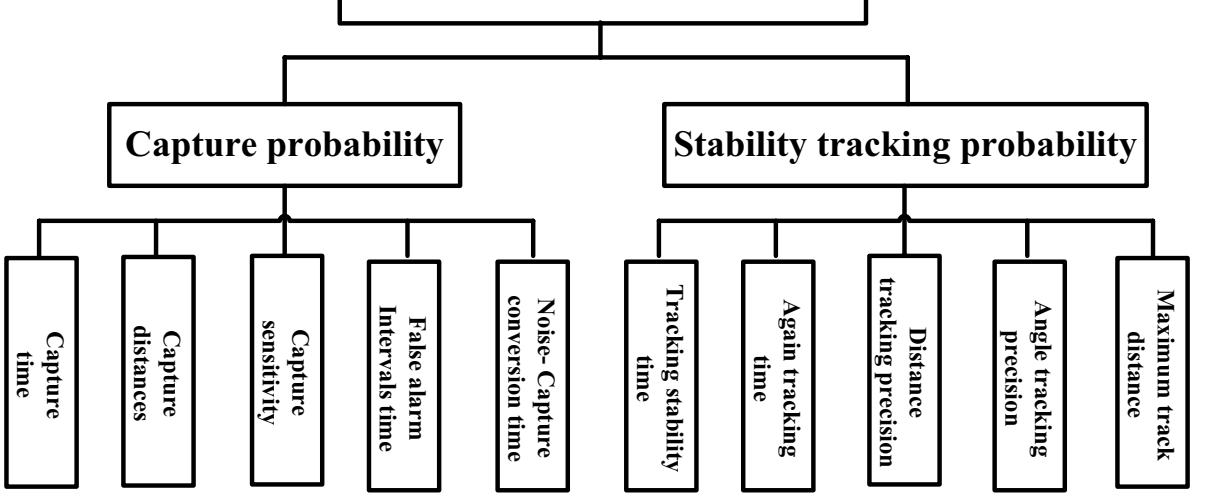


$\frac{\delta L}{\delta \omega}=\sum_{j=1}^{m} d_{j}+\xi * \omega_{j}=0$.

After calculating formula (7) and $\sum_{j=1}^{m} \omega_{j}^{2}=1$, we can obtain normalized $\omega_{j}=d_{j} / \sum_{j=1}^{m} d_{j}$.

\section{Key problems in information fusion}

Two key problems need to be solved in the application of multi-source information fusion method. One is the information consistency test, which answers the question whether the information fusion process can be carried out. The other is to determine information weight, which answers the question how to carry out information fusion. In the radar seeker fusion evaluation process, referring to Bayes theory, we regard the evaluation indexes which are derived from test different test stages data as random variables, such as angle measurement accuracy and detection probability. Then the methods of information consistency test and weight determination are discussed.

\subsection{Information consistency test}

Only when information consistency checking has been done can information fusion be carried out in the next step, otherwise information fusion will cause wrong result and lose its significance. Simple data consistency testing methods include mean test, median test, etc. The specific method is to take the deviation between sample data and sample mean or median as reference. When the deviation is below the given threshold, the consistency checking is considered successful and sample data is effective and available. When the deviation exceeds the threshold, the sample data is considered abnormal and need to be eliminated to ensure data consistency. This data consistency testing method is simple and practical. However, it can only be applied to large sample data without considering the distribution of test samples. When the test data itself has certain time-varying characteristics, this method may bring some wrong conclusions.

Information consistency test can be divided into distribution test and parameter test according to whether test quantity distribution model is known. When the test sample is large enough, Pearson $\chi^{2}$ Test is a common test method. When test sample is insufficient, consistency test methods include Rank Sum Test and Information measure.

\section{Rank Sum Test}

Taking detection probability $\theta$ as an example, we use Rank Sum Test method to complete distribution test for $\theta$ considering that radar seeker evaluation test sample is small and $\theta$ distribution model is not known.

Definition For $X\left(x_{1}, x_{n}, \ldots x_{n}\right)$, arranging its sample element $x_{i}$ in order from small to large we can get a new sequence $X^{*}\left(x_{1}^{*}, x_{2}^{*}, \ldots x_{n}^{*}\right)$. If $x_{i}=x_{k^{\prime}}^{*}$ then $k$ is the rank for $x_{i}$, remember as $r_{k}\left(x_{i}\right)$.

For two (or more) elements $x_{j}$ and $x_{j+t}$ with same value, their rank are same, that is $r_{k}\left(x_{j}\right)=r_{k}\left(x_{j+t}\right)$, which are the smaller order in sequence $X^{*}$.

Let $Y^{i}\left(y_{1}^{i}, y_{2}^{i}, \ldots y_{n_{i}}^{i}\right)$ and $Y^{j}\left(y_{1}^{j}, y_{2}^{j}, \ldots y_{n_{j}}^{j}\right)$ be two group test samples, we use Rank Sum Test method to ensure whether $Y^{j}$ is consistent with $Y^{i}$. Mixing elements in both $Y^{j}$ and $Y^{i}$ from small to large in order to get new sample $Y^{*}\left(y_{1}^{*}, y_{2}^{*}, \ldots y_{n_{i}+n_{j}}^{*}\right)$, we regard rank sum for $Y^{j}$ in $Y^{*}$ as:

$T\left(Y_{j}\right)=\sum_{j=1}^{n_{j}} r_{k}\left(y_{j}\right)$.

The principle of Rank Sum Test is that when $Y^{j}$ and $Y^{i}$ are subject to the same distribution model, $T\left(Y_{j}\right)$ will be in a reasonable range rather than too big or too small. When confidence level $a$ is given, we can use Rank Sum Test Table to judge whether $Y^{j}$ is consistent with $Y^{i}$.

\section{Information Measure}

The notion of Shannon entropy was firstly introduced as an uncertainty measure for discrete distributions in information theory and it is developed in the contexts of statistical mechanics and system features. For a continuous random variable $X$ with density function $f(x)$ and distribution function $F(x)$, The entropy $H(X)$ is defined as:

$H(X)=-E[\log f(X)]=-\int \log f(x) d F(x)$.

Note that entropy is a function of the distribution. It does not depend on the actual values but only on the probabilities of $X$.

The Kullback-Leibler divergence, also known as relative entropy, is an information measure between two probability density functions $f(x)$ and $g(x)$. It is defined as

$I(f, g)=\int \log \frac{f(x)}{g(x)} d F(x)=H(G)-H(F)$.

From the point of view of information entropy, $I(f, g)$ describes the similarity degree of two probability distributions, which is the basis of consistency test. $I(f, g)$ does not have some properties of distance measure and cannot be used directly as distance measure, so it needs to be adjusted. Distance measure should satisfy some 
properties. To obtain a rigorous information distance measure, we adjust $I(f, g)$ to $D I(f, g)$, which is defined as:

$D I(f, g)=\left|\int g(x) \log \frac{f(x)}{g(x)} d F(x)\right|$.

$D I(f, g)$ satisfies following properties:

1. $D I(f, g)=D I(g, f)$.

2. $D I(f, g) \geq 0$. If and only if $f=g, D I(f, g)=0$.

Based on the definition of information distance measure $D I(f, g)$, as long as the appropriate threshold $\Delta$ is set, the consistency test of different sample information can be completed by comparing the relation $D I(f, g)$ and $\Delta$.

We conclude the effectiveness of Kullback-Leibler information measure by presenting an example due to normal random variables.

Considering random variables $X$ and $Y$ both subject to standard normal distribution, the joint density function for the pair of random variables $(X, Y)$ with the coefficient correlation $\rho$ is given by,

$f(x, y)=\frac{1}{2 \pi \sqrt{1-\rho^{2}}} \exp \left[\frac{-\left(x^{2}-2 \rho x y+y^{2}\right)}{2\left(1-\rho^{2}\right)}\right]$.

where $(x, y) \in R^{2}$ and $|\rho| \leq 1$. Taking all these assumptions into account, we can conclude that the conditional random variable $X \mid Y$ is subject to the normal distribution $N\left(\rho Y,\left(1-\rho^{2}\right)\right)$. By applying some simple computation for normal distribution with mean $\mu$ and variance $\sigma^{2}$, we can obtain,

$H(X)=\log \sqrt{2 \pi \sigma^{2}}+\frac{1}{2}$.

Considering that entropy does not depend on mean parameters, we can obtain,

$H(X \mid y)=\log \sqrt{2 \pi\left(1-\rho^{2}\right)}+\frac{1}{2}$.

$\int f(x \mid y) \log f(x) d x=-\log \sqrt{2 \pi}-\frac{1}{2}\left(1-\rho^{2}+y^{2} \rho^{2}\right)$.

$I\left(f_{X \mid y}, f_{X}\right)=\frac{1}{2} \rho^{2}\left(y^{2}-1\right)-\log \sqrt{1-\rho^{2}}$.

It can be seen from the Figs. 7 and 8 that the information measure $I\left(f_{X \mid Y}, f_{X}\right)$ increases with the increase of correlation coefficient $\rho$. When the correlation coefficient is fixed and the variables $Y$ increase, the information measure between conditional distribution variables $X \mid Y$ and variables $X$ also increases. These two trends are consistent

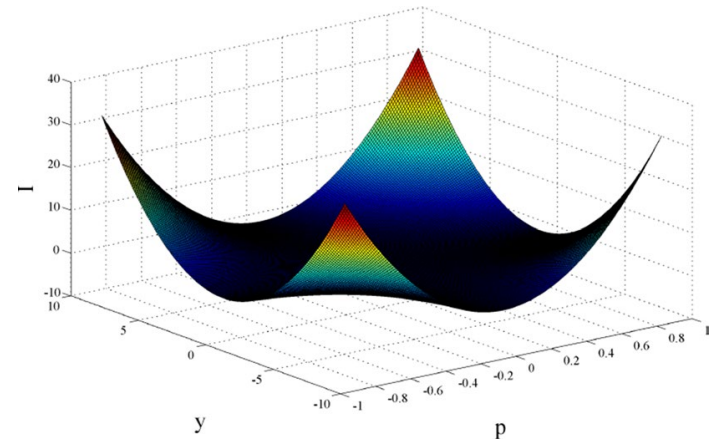

Fig. $7 I\left(f_{X \mid Y^{\prime}} f_{X}\right)$ simulation

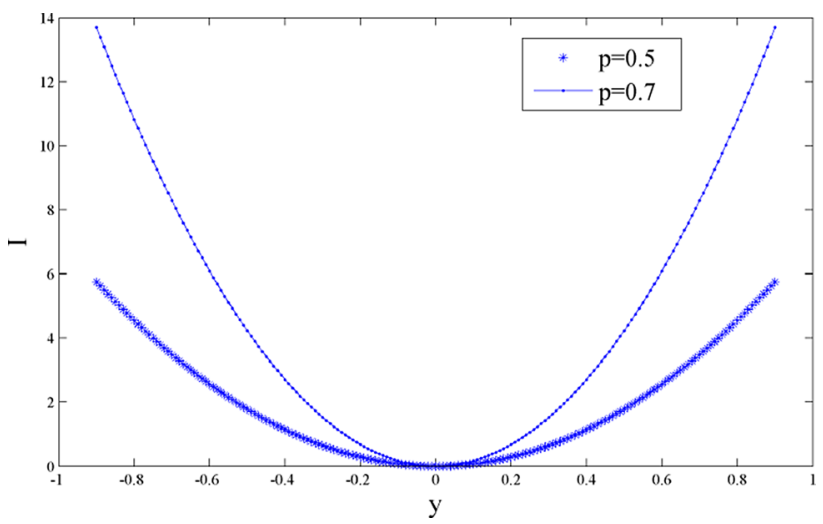

Fig. $8 I\left(f_{X \mid y^{\prime}} f_{X}\right)$ simulation when $\rho$ is fixed

with the expectant situation, which proves the effectiveness of the method.

Compared with the two methods, the advantage of rank sum test is that the operation is intuitive. It does not need to establish the distribution model of samples in advance and it has wide applicability. The advantage of information measure test lies in that the test conclusion obtained from sample information is more objective.

\subsection{Information weight determination method}

Distributing appropriate weights to different information is the second key part of the information fusion process. When multi-source information passes the consistency test, reasonable weight is very important for reliable decision-making conclusions. In the information fusion evaluation of angle measurement, we take the field test sample as a reference, usually take its weight as unit 1 , and then examine the relative weights of simulation samples. This part introduces PLT criterion and analyses its effectiveness in information weight allocation.

The test data obtained in different test stages have different characteristics, which has been studied in literature 
Table 1 Information weight obtained by PLT

\begin{tabular}{llllllll}
\hline$n_{0}$ & 10 & 20 & 50 & 100 & 200 & 500 & 1000 \\
\hline$a_{i}^{*}$ & 0.006 & 0.049 & 0.097 & 0.127 & 0.166 & 0.289 & 0.349 \\
\hline
\end{tabular}

[26]. These characteristics should been reflected in information weight in fusion evaluation process. To allocate information weight, Ibrahim proposed Penalized Likelihood-type Criterion. $X$ is field test samples, $X_{i}$ is historical test samples, $n_{i}$ is sample capacity, $\pi_{i}$ is prior distribution for $X$. Let

$\inf G\left(a_{i}\right)=-2 \log h^{*}\left(a_{i}\right)+\frac{\log n_{i}}{a_{i}}$.

$h^{*}\left(a_{i}\right)=\int_{\theta \in \Theta} L(\theta \mid X) L\left(\theta \mid X_{i}\right)^{a_{i}} \pi_{i}(\theta) d \theta$

$a_{i}^{*}$ is the weight for $X_{i}$ under PLT criterion. In the process of radar seeker performance test and evaluation, the historical sample size is often larger than the field sample size. Under this condition, we expect to prevent the scene sample from being flooded by reducing the historical sample weight. The sample weights determined by PLT criterion are in line with this requirement. We validate this view by interpreting simulation data.

Let $X$ whose sample size is $n=100$ and $X_{i}$ whose sample size is $n_{i}$ be mutual independence and subject to normal distribution $N(\theta, 1)$. Regardless of the prior distribution $\pi_{i}(\theta)$ for $\theta$, the historical samples weight obtained according to PLT criterion is shown in the Table 1.

\section{Example}

\subsection{Single parameter fusion estimation}

In this part, we illustrate the implementation process and effectiveness of radar seeker angle measurement performance based on information fusion through test data. Radar seeker uses mono-pulse angle measurement in both hardware-in-the-loop and field testing phases.

The 100 hardware-in-the-loop simulation test data and 10 flying test data for the same radar seeker angle measurement performance are shown in Fig. 9.

The consistency test results of the test data are shown in Table 2. The weight of the hardware-in-the-loop simulation test data calculated by PLT criterion are 0.318 .

The performance evaluation results of hardware-inthe-loop simulation, field test and fusion evaluation are compared as shown in Table 3.
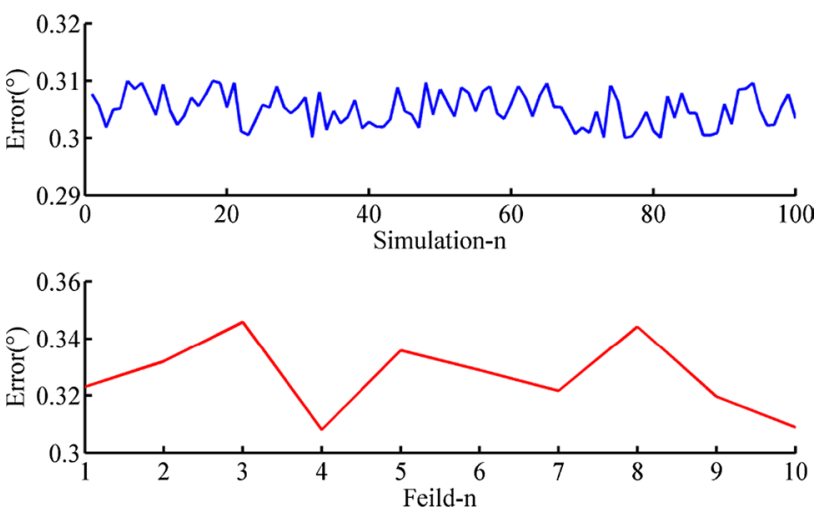

Fig. 9 Simulation test data and fling test data

Table 2 Consistency test result

\begin{tabular}{lll}
\hline Test method & Rank sum test $(\alpha=0.05)$ & K-L measure $(\Delta=0.90)$ \\
\hline Test result & Pass & Pass \\
\hline
\end{tabular}

Table 3 Angle measurement error

\begin{tabular}{lll}
\hline Simulation $\left({ }^{\circ}\right)$ & Flying test $\left(^{\circ}\right)$ & Fusion evaluation $\left(^{\circ}\right)$ \\
\hline 0.305 & 0.322 & 0.317 \\
\hline
\end{tabular}

\subsection{Multi parameter fusion evaluation}

To further evaluate the anti-jamming performance of radar seekers, we carried out performance test scheme based on active homing radar seeker and high-power active suppression jamming equipment. Then we construct a common index system referring to Radar Test Outline, as shown in Fig. 6. As the same as the angle measurement accuracy in part $A$, other indicators are tested for information consistency. The results show that these indicators meet the requirements of information consistency and can be carried out in information fusion. The first column of Table 4 is corresponding to index system in Fig. 6. Where $x_{1}$ indicates capture time and $x_{10}$ indicates maximum track distance. Due to the particularity of experimental data, we need to preprocess these data. Considering performance design index as benchmark, the relative index attribute values in simulation and flying test are listed in the second column and the third column of Table 4 . The fourth column of Table 4 is index fusion result. It is calculated by weighted sum of simulation data and flying test, where the 
Table 4 Performance fusion evaluation

\begin{tabular}{lllll}
\hline & Simulation & Flying test & Fusion result & Index weight \\
\hline$x_{1}$ & 1.02 & 1.08 & 1.061 & 0.06 \\
$x_{2}$ & 0.94 & 0.91 & 0.920 & 0.08 \\
$x_{3}$ & 1.17 & 0.89 & 0.979 & 0.07 \\
$x_{4}$ & 1.22 & 0.82 & 0.947 & 0.12 \\
$x_{5}$ & 0.82 & 0.88 & 0.861 & 0.09 \\
$x_{6}$ & 1.11 & 1.23 & 1.192 & 0.09 \\
$x_{7}$ & 0.82 & 0.92 & 0.888 & 0.13 \\
$x_{8}$ & 1.19 & 1.17 & 1.176 & 0.11 \\
$x_{9}$ & 0.93 & 0.8 & 0.841 & 0.12 \\
$x_{10}$ & 0.87 & 0.93 & 0.911 & 0.13 \\
\hline
\end{tabular}

weight of the hardware-in-the-loop simulation test data calculated by PLT criterion are 0.318 . The fifth column of Table 4 is index weight calculated by maximization-deviation method.

It can be seen from Tables 3 and 4 that the radar seeker performance fusion evaluation results retain the overall trend of outfield test results. Besides, fusion evaluation method can use the advantages of abundant test data samples in hardware-in-the-loop simulation phase to improve the uncertainty caused by insufficient field test samples. This result shows that the fusion test method is objective and effective. The final performance evaluation result obtained by weighted sum method is:

$s=x * w^{\top}$.

The calculation result is 0.968 , which is the basis for further comparing the relative performance of different radar seeker. By comparing the performance scores $s$ of different seeker, we can get their relative advantages and disadvantages.

Compared to literature [26], this paper focuses on two new key issues in the process of radar performance fusion evaluation. The first one is information consistency test method and the second one is information weight empowerment. The results are of great significance to the improvement of performance fusion evaluation.

\section{Conclusion}

Considering radar seeker angle measurement performance evaluation as a background, an evaluation method based on information fusion is proposed in this work. Consistency between different information is a prerequisite in the application process of fusion evaluation method. We first solve the problem of consistency checking among different information using Rank Sum method and K-L information distance measurement. The second subject concerns with information elements weight empowerment method in the multi-source information-fusion evaluation. Penalized Likelihood-type Criterion is a suitable weight-adjustment model where the repeatability of information in different test stages can be used as an adjustment factor to obtain more realistic evaluation results. The adjusted information weights conform to the actual assessment requirements. We use both simulation data and field test data to verify the effectiveness of performance fusion evaluation method in the simulation verification part. The radar angle measurement performance evaluation results that we obtained are objective and rational in Example A. Using the method of weighted summation, we get the comprehensive evaluation results of radar seeker under multi index system in Example B. Both two experimental results can show the effectiveness of information fusion evaluation method we proposed in this paper.

Acknowledgements The authors thank all the reviewers and editors for their valuable comments and works. This paper is supported by the National Natural Science Foundation of China (No. 61501471).

\section{Compliance with ethical standards}

Conflict of interest The authors declare that they have no conflict of interest.

\section{References}

1. Baker CJ, Alsaif S, Smith GE (2014) Echoic flow for cognitive radar guidance. In: Radar conference, pp 0490-0495

2. Hong L, Dai FZ, Wang XL (2018) Knowledge-based wideband radar target detection in the heterogeneous environment. Signal Process 8:169-179

3. Edrich M, Schroeder A, Meyer F (2014) Design and performance evaluation of a mature FM/DAB/DVB-T multi-illuminator passive radar system. Radar Sonar Navig 8:114-122

4. Marin M, Craciun EM (2017) Uniqueness results for a boundary value problem in dipolar thermo elasticity to model composite materials. Compos Part B Eng 126:27-37

5. Marin M, Baleanu D (2017) Effect of micro temperatures for micro polar thermo elastic bodies. Struct Eng Mech 61(3):381-387

6. Wang N, Sun JP (2017) Netted radar management based on anti-jamming capability. In: 20th international conference on information fusion, pp 1912-1918

7. Chang H, Xia R (2014) Performance evaluation of interferometric synthetic aperture radar jamming. Electron Sci Technol 19:121-127

8. El Mashade MB (2013) Analytical performance evaluation of adaptive detection of fluctuating radar targets. Radio Electron Commun Syst 56:321-334

9. Khawar A, Abdelhadi A (2015) Target detection performance of spectrum sharing MIMO radars. IEEE Sens J 15:4928-4940

10. Ali MR (2019) A truncation method for solving the time-fractional Benjamin-Ono equation. J Appl Math 18:1-7 
11. Ali MR, Ma W-X (2019) New exact solutions of nonlinear $(3+$ 1)-dimensional Boiti-Leon-Manna-Pempinelli equation. Adv Math Phys 2019:9801638. https://doi.org/10.1155/2019/98016 38

12. Yang $Y$, Xiao SP (2018) Performance analysis of radar detection for correlated gamma fluctuating targets in $\mathrm{K}$ distributed sea clutter. Digital Signal Process 79:136-141

13. He Y, Xiu JJ, Guan X (2016) Evaluation of radar data processing performance. In: Radar data processing with applications, vol 18 , pp 427-440

14. Wen ZZ, Wang XS (2015) Radar target recognition. Higher Education Press, Beijing

15. Gong T, Yan H (2016) Multi-sensor information fusion and application. Appl Mech Mater 32:2623-2626

16. Bowman C (2005) Unifying data fusion and resource management software development approaches using the dual node network architecture. In: Unification of fusion theories

17. Gong T, Yan H (2016) Multi-sensor information fusion and application. Appl Mech Mater 32:2623-2626

18. Catano V, Gauger J (2017) Information fusion: intelligence centers and intelligence analysis. Inf Shar Mil Oper 17:17-34

19. Ali MR, Mousa MM, Ma W-X (2019) Solution of nonlinear Volterra integral equations with weakly singular kernel by using the HOBW method. Adv Math Phys 2019:1705651. https://doi. org/10.1155/2019/1705651

20. Xu Z, Zhao N (2016) Information fusion for intuitionistic fuzzy. Inf Fus 28:10-23
21. George DE (2016) On the divergence of information filter for multi sensors fusion. Inf Fus 27:76-84

22. Wei P, Ball JE, DerekT (2016) Multi-sensor conflict measurement and information fusion. In: Proceedings of the SPIE, vol 9842

23. Yu SW, Huang TZ (2017) Exponential weighted entropy and exponential weighted mutual information. Neurocomputing 22:86-94

24. Salimeh S (2015) Bayesian weighted information measures. Interdiscip Bayesian Stat 275-289

25. Eftekhari A, Yang D, Wakin MB (2018) Weighted matrix completion and recovery with prior subspace information. IEEE Trans Inf Theory 64:4044-4071

26. Liu XH, Yang J (2019) Weight empowerment method in information fusion for radar-seeker performance evaluation. J Eng 12:8452-8457

27. Chen $B$, Jin $T$ (2015) Building interior structure reconstruction from through-the- wall radar image using MST-based method. Acta Electron Sin 43:1682-1688

28. An T, Sai Z (2018) An overview of radar clutter simulation. In: IOP conference series: materials science and engineering, p 042198

Publisher's Note Springer Nature remains neutral with regard to jurisdictional claims in published maps and institutional affiliations. 